\title{
PLASMA ENHANCED ATOMIC LAYER DEPOSITION OF SOLID-STATE ELECTROLYTE LI-TA-O FOR SOLID-STATE BATTERIES
}

\author{
${ }^{1}$ Pavel FEDOROV, ${ }^{1,2}$ Denis OLKHOVSKII, ${ }^{1,3}$ Denis NAZAROV, ${ }^{1}$ Vladislav CHERNYAVSKY, \\ ${ }^{1,4}$ Yury KOSHTYAL, ${ }^{4}$ Aleksander RUMYANTSEV, ${ }^{1}$ Anatoly POPOVICH, ${ }^{1 *}$ Maxim Yu. MAXIMOV \\ ${ }^{1}$ Peter the Great Saint-Petersburg Polytechnic University, Saint Petersburg, Russia, \\ fedorovpave199@yandex.ru, Odl-ne@yandex.ru,dennazar1@ya.ru, vlad.dixdr@gmail.com, \\ yury.koshtyal@gmail.com, rumyantsev.amr@gmail.com, director@immet.spbstu.ru, \\ ${ }^{1 *}$ Correspondence: maximspbstu@mail.ru \\ ${ }^{2}$ Saint-Petersburg State Institute of Technology, Saint Petersburg, Russia \\ ${ }^{3}$ Saint Petersburg State University, Saint Petersburg, Russia \\ ${ }^{4}$ loffe Institute, Saint Petersburg, Russia
}

https://doi.org/10.37904/nanocon.2021.4336

\begin{abstract}
Solid-state batteries (SSBs) are regarded as the next step in energy storage technology. One of the main problems in developing such batteries is developing and synthesizing solid-state electrolytes (SSE). The main factor that stops the introduction of SSBs into everyday life is the low ionic conductivity of Li ions in modern SSEs. Therefore, the primary purposes of the work are to establish the reasons for the manifestation of this inhibiting factor and to assess the possibility of using new materials for SSLIB. Solution these problems ultimately can give an impetus to the development and promotion of such type batteries. In this work, SSE was obtained by the atomic layer deposition (ALD) method, allowing the formation of homogeneous coatings with precision control of the thickness. ALD of LixTaOy thin films on silicon and stainless steel substrates using $\mathrm{Ta}(\mathrm{OEt}) 5$ and LiOtBu was studied. The synthesis temperature was $300{ }^{\circ} \mathrm{C}$, which is based on the previous research. Samples were synthesized with different ratios of $\mathrm{Li}: \mathrm{Ta}=1: 2,1: 3,1: 7$, and depending on the ratio of metals, the growth rate per supercycle varied from 0.21 to $0.46 \mathrm{~nm}$. The film thickness was determined by spectral ellipsometry. A scanning electron microscopy was used to determine the conformity and morphology of the coatings. X-ray Photoelectron Spectroscopy was used to determine the elemental composition on the surface and in the bulk of SSE. According to X-ray diffraction, thin films are amorphous. Cyclic voltammetry has been used to study how thin films will respond to different voltages. The cathodic region cycling at various discharge currents (from 20 to $80 \mu \mathrm{A} / \mathrm{cm} 2$ ) presents a low capacity.
\end{abstract}

Keywords: Atomic layer deposition, lithiated tantalum oxide, solid-state electrolyte, thin films, solid-state batteries

\section{INTRODUCTION}

Lithium-ion batteries (LIBs), due to their unique characteristics (specific energy, specific power, wide operating temperature range, recharge resource, etc.), have already taken root in many areas of human activity, from industrial technology to household and portable appliances [1]. But due to the use of a liquid organic electrolyte in LIBs, they are characterized by the following disadvantages: first of all, we need to introduce additional process - formation, which complicates the operation of their production, and the second drawback is the decrease in output parameters and maximum capacity with use due to the formation of solid-electrolyte interphase (SEI) $[2,3]$. Therefore, today in lithium-ion energy, quite a lot of research is devoted to solid-state batteries (SSBs). This is because they use a solid inorganic compound instead of the usual liquid electrolyte 
[4]. Just because of this, the main factor that distinguishes SSBs from ordinary LIBs has increased levels of fire and explosion safety due to a decrease in the proportion of combustible material. So it becomes possible to introduce lithium-ion energy in new promising areas, such as electric vehicles.

Compounds similar in structure to LISICON (Li Super lon CONductors) [5] are chosen as a solid electrolyte: $\mathrm{Li}_{1+x} \mathrm{Ti}_{2-x} \mathrm{M}_{\mathrm{x}}\left(\mathrm{PO}_{4}\right)_{3}(\mathrm{M}=\mathrm{Ge}, \mathrm{Ti}, \mathrm{Sn}, \mathrm{Zn}, \mathrm{Hf}[5]), \mathrm{Li}_{2} \mathrm{~S}-\mathrm{P}_{2} \mathrm{~S}_{5}$, and others [6]. As specific examples, we can cite studies of structures: $\mathrm{Li}_{x} \mathrm{La}_{3} \mathrm{Zr}_{2} \mathrm{O}_{12}, \mathrm{Li}_{1+x} \mathrm{Al}_{x} \mathrm{Ge}_{2-x}\left(\mathrm{PO}_{4}\right)_{3}, \mathrm{Li}_{10} \mathrm{GeP}_{2} \mathrm{~S}_{12}$, etc. $[1,7,8,9]$. The standard method for obtaining these structures is the sol-gel method $[5,6]$. Still, the growing popularity of synthesis using atomic layer deposition (ALD) can be considered a progressive and better method for obtaining solid electrolytes. The most important advantages of ALD over the sol-gel method, in this case, can be called: obtaining conformal coatings, obtaining the required thickness with a minimum error, and the possibility of covering complex geometric shapes $[1,10]$. Less important, but still advantages can be called: the repeatability of the process, its self-limiting, and synthesis at relatively low temperatures $[10,11,12]$.

This article describes the results of studies of obtained $\mathrm{LiTaO}$ thin films on steel and silicon substrates by the ALD method. We studied samples with the ratio $\mathrm{Li}: \mathrm{Ta}=1: 2,1: 3$, and 1:7, their growth rate on the substrate, and the elemental composition in-depth. Based on which the optimal Li-Ta-O films were selected for subsequent syntheses on the cathode material [13].

\section{MATERIALS AND METHODS}

The deposition of $\mathrm{Li}-\mathrm{O}$ and $\mathrm{Ta}-\mathrm{O}$ for $\mathrm{Li}-\mathrm{Ta}-\mathrm{O}$ films was carried out by ALD with Picosun R-150 setup at $300{ }^{\circ} \mathrm{C}$ at a base pressure of 8-12 hPa. Lithium tert-butoxide (LiOtBu, $97 \%$, Sigma Aldrich) and tantalum(V) ethoxide $\left(\mathrm{Ta}(\mathrm{OEt})_{5}, 99,98 \%\right.$ Sigma-Aldrich), were used as metal-containing reagents. Remote oxygen plasma $\left(\mathrm{O}_{2}-\mathrm{p}\right)$ was applied as a counter-reagent.

Precursors' containers were heated to $190{ }^{\circ} \mathrm{C}$ for $\mathrm{Ta}(\mathrm{OEt})_{5}$ and $220{ }^{\circ} \mathrm{C}$ for LiO'Bu. All depositions were performed as follows. After pulsing of metal-containing reagent $\left(\mathrm{Ta}(\mathrm{OEt})_{5}-1.5 \mathrm{~s}\right.$ or $\left.\mathrm{LiO}^{\dagger} \mathrm{Bu}-3.0 \mathrm{~s}\right)$ the excess of the precursor was purged with nitrogen $(99.999 \%)$ within 10 s. Pulse times for $\mathrm{O}_{2}$ plasma was 10 s. For deposition of $\mathrm{Li}-\mathrm{Ta}-\mathrm{O}$ thin films we used super cycles consisting of one $\mathrm{LiO}^{\mathrm{t}} \mathrm{Bu} / \mathrm{O}_{2}-\mathrm{p}$ pulses and two, three or seven $\mathrm{Ta}(\mathrm{OEt})_{5} / \mathrm{O}_{2}-\mathrm{p}$ pulses: $\left[\mathrm{LiO}^{\mathrm{t}} \mathrm{Bu} / \mathrm{O}_{2}-\mathrm{p}\right]+\left[\mathrm{Ta}(\mathrm{OEt})_{5} / \mathrm{O}_{2}-\mathrm{p}\right]^{*} 2=\mathrm{LiTaO}-1 / 2, \quad\left[\mathrm{LiO} \mathrm{Bu} / \mathrm{O}_{2}-\mathrm{p}\right]+\left[\mathrm{Ta}(\mathrm{OEt})_{5} / \mathrm{O}_{2}-\right.$ $\mathrm{p}]^{*} 3=\mathrm{LiTaO}-1 / 3,\left[\mathrm{LiO}^{*} \mathrm{Bu} / \mathrm{O}_{2}-\mathrm{p}\right]+\left[\mathrm{Ta}(\mathrm{OEt})_{5} / \mathrm{O}_{2}-\mathrm{p}\right]^{*} 7=\mathrm{LiTaO}-1 / 7$. To obtain the required films, 100-150 synthesis supercycles were performed. Estimated growth rates for Ta-O was $0.05 \mathrm{~nm} /$ cycle, for $\mathrm{Li}-\mathrm{O}-0.11$ $\mathrm{nm} /$ cycle.

Monocrystalline silicon wafers (surface orientation 100, the size $4 \times 4 \mathrm{~cm}$, Telecom-STV Co., LTD, Zelenograd, Moscow, Russia) and stainless-steel plates (316SS, Tob New Energy Technology Co., LTD, diameter $15.8 \mathrm{~mm}$ ) were used as substrates.

The thicknesses of the films were measured by spectral ellipsometry (wavelength range $350-1000 \mathrm{~nm}$ ) using Ellips-1891 SAG ellipsometer (CNT, Novosibirsk, Russia). The morphology of the films was studied by scanning electron micrographs of flat and cross-sections were obtained by a Supra 55 VP scanning electron microscope (SEM, Zeiss, Oberkochen, Germany). X-ray diffraction (XRD) studies were performed using a Bruker D8 ADVANCE (Cu-Ka). The obtained results were processed by the Rietveld method using TOPAS 5 software (XRD). Finally, the chemical composition of the films was studied by X-ray photoelectron spectra (XPS) and was obtained on an Escalab 250Xi spectrometer (Thermo Fisher Scientific, Waltham, MA, USA).

Electrochemical studies were performed in CR2032 coin cells, the samples of $\mathrm{Li}-\mathrm{Ta}-\mathrm{O}$ were deposited on the 316SS surface. Lithium foil, polyolefin porous film 2325 (Celgard, Charlotte, NC, USA), and TC- E918 (Tinci, Guangzhou, China) solution were used as the counter electrode, separator, and electrolyte, respectively. The coin cells (CR2032) were assembled in OMNI-LAB (VAC) glove box under argon atmosphere. Cyclic voltammetry (CV) was performed using a potentiostat PGSTAT302N+ (Autolab, Utrecht, the Netherlands) in the range of $0.1-4.3 \mathrm{~V}$ with a scan rate of $0.5 \mathrm{mV} / \mathrm{s}$. Cyclic charge/discharge was performed using the battery 
testing system CT-3008W-5V10mA (Neware, Shenzhen, China) at room temperature in the range of voltage 3.0-4.3 V, at current $20-80 \mu \mathrm{A} / \mathrm{cm}^{2}$.

\section{RESULTS AND DISCUSSIONS}

\subsection{Synthesis of Li-Ta-O structures}

Previous experiments with the synthesis of tantalum structures as thin films $[1,14]$ made it possible to select the conditions for obtaining the $\mathrm{Li}-\mathrm{Ta}-\mathrm{O}$ system; the corresponding synthesis parameters are presented in the Materials and Methods section.

A series of experiments were carried out to obtain $\mathrm{Li}-\mathrm{Ta}-\mathrm{O}$ with the ratios of the pulse $\mathrm{LiO} \mathrm{Bu} / \mathrm{Ta}(\mathrm{OEt})_{5}$ in the supercycle: 1:2, 1:3, and 1:7. The ratios were selected based on research results published elsewhere [15]. As a result, the investigated growth rate of $\mathrm{LiTaO}-1 / 2$ corresponds to the expected $-0.21 \mathrm{~nm} /$ supercycle (Table 1). According to spectral ellipsometry, the samples $\mathrm{LiTaO}-1 / 3$ and $\mathrm{LiTaO}-1 / 7$ have a higher growth rate than expected, possibly caused by the stimulation of growth with an increase in the number of pulse $\mathrm{Ta}(\mathrm{OEt})_{5}$.

Table 1 The thicknesses of the Li-Ta-O films measured using SEM and ellipsometry and the growth parameters of the films according to the ellipsometry data

\begin{tabular}{|c|c|c|c|c|c|}
\hline $\begin{array}{c}\text { Samples - } \\
\text { Li/Ta ratio }\end{array}$ & $\begin{array}{c}\text { SEM average } \\
\text { thickness, } \mathbf{n m}\end{array}$ & $\begin{array}{c}\text { Ellipsometry } \\
\text { average } \\
\text { thickness, } \mathbf{n m}\end{array}$ & $\begin{array}{c}\text { Number of } \\
\text { supercycles }\end{array}$ & $\begin{array}{c}\text { Growth rate, } \\
\text { nm/supercycle }\end{array}$ & $\begin{array}{c}\text { Estimated growth } \\
\text { rate, nm/supercycle }\end{array}$ \\
\hline $\mathrm{LiTaO}-1 / 2$ & 45.05 & 30.9 & 150 & 0.21 & 0.21 \\
\hline $\mathrm{LiTaO}-1 / 3$ & 39.44 & 37.9 & 120 & 0.32 & 0.26 \\
\hline $\mathrm{LiTaO}-1 / 7$ & 58.75 & 62.61 & 100 & 0.63 & 0.46 \\
\hline
\end{tabular}

The thicknesses measured by SEM (Table 1) for the LiTaO-1/3 and LiTaO-1/7 samples are close to the data obtained by ellipsometry. For LiTaO-1/2, there is a discrepancy between the two measurement methods. This may be due to the film thickness gradient and edge effects when measuring thin films on SEM. According to the SEM micrograph (Figure 1), the surface of $\mathrm{LiTaO}-1 / 7$ is homogeneous without visible defects.

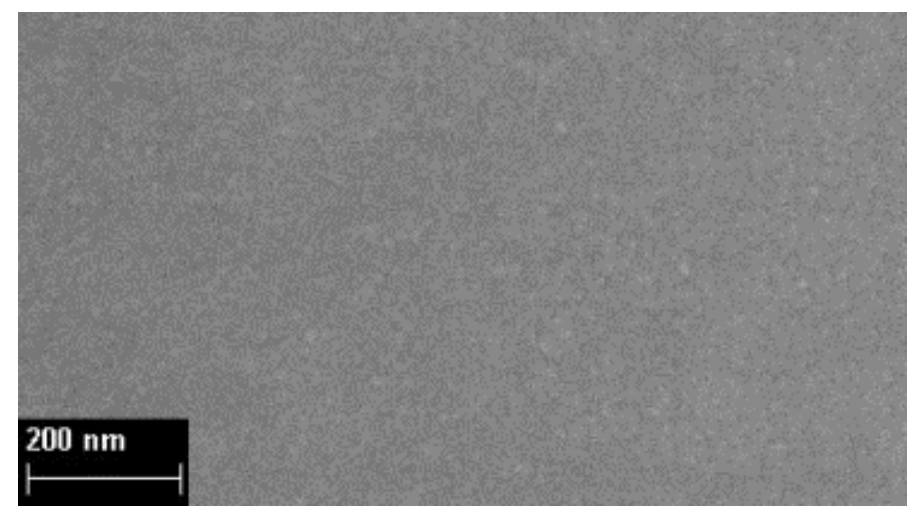

Figure 1 SEM image demonstrates a LiTaO-1/7 coating sample on the silicon substrate

For a detailed study of the chemical composition of the Li-Ta-O system, studies were carried out by the XPS method (LiTaO-1/7 - Table 2). As a result, the composition of the samples' surface and the bulk of the coating were investigated before and after etching the surface layer with argon ions (90 and $300 \mathrm{sec}, 500 \mathrm{eV})$. As a result, the following elements were found on the sample's surface: lithium, tantalum, oxygen, and carbon (Table 2). Also, by the etching proceeds, it can be seen the number of lithium decreases and the proportion 
of tantalum increases. This may be due to the presence of a gradient of elements in the bulk of the film. Thus, the surface is enriched with lithium, while tantalum is found mainly in the bulk of the film.

According to the CIL peaks of carbon (Figure 2), before etching, $\mathrm{C}$ is present on the surface in the composition of compounds, including the $\mathrm{C}-\mathrm{OH}, \mathrm{C}-\mathrm{C}, \mathrm{C}-\mathrm{H}$ groups, and $\mathrm{C}=\mathrm{O}$ bonds (peak shoulder at $284.8 \mathrm{eV}$ ) and $\mathrm{COOH}$ (289 eV peak). After $90 \mathrm{sec}$ etching of the surface layer, the carbon signal has dropped noticeably, which indicates its absence in bulk (concentration in the analysed sample is below the detection threshold).

The spectra of Li1s (Figure 2), obtained without preliminary etching and after 90 and $300 \mathrm{sec}$ etching, exhibit a broad maximum in the region of manifestation of lithium compounds. In the region of the location of the maximum, bands appear that characterize the presence of lithium in various compounds: $\mathrm{Li}_{2} \mathrm{O}, \mathrm{Li}_{2} \mathrm{CO}_{3}, \mathrm{LiOH}$. The low intensity and signal/interference ratio of the Li1s spectra do not allow one to reveal the preferred state. After etching, the intensity of the peak drops by almost a factor of two.

The position of the maxima in the Ta4f spectrum (Figure 2) before etching corresponds to the $\mathrm{TaO}_{2}$ compound, which is inconsistent with the data obtained from the $\mathrm{O} 1 \mathrm{~s}\left(\mathrm{Ta}_{2} \mathrm{O}_{5}\right)$ spectrum. This discrepancy can be caused by the high described difficulties and errors in the calibration of the position of the peaks (charge compensation). Nevertheless, in the spectra after 90 sec etching, the appearance of new peaks (components) in the region of lower energies is noticeable, these peaks correspond to the $\mathrm{Ta}^{0}$ and $\mathrm{Ta}^{2+}$ states of tantalum. Therefore, an unambiguous conclusion can be made about reducing tantalum (possibly even to metallic $\mathrm{Ta}$ ) during etching.

Table 2 Element ratios before and after etching a Li-Ta-O-1/7 film

\begin{tabular}{|c|c|c|c|}
\hline Element & Before etching (at. \%) & After etching 90 s (at. \%) & After etching 300 s (at. \%) \\
\hline $\mathrm{O}$ & 47.65 & 55.29 & 3.15 \\
\hline $\mathrm{Ta}$ & 12.45 & 31.94 & 1.15 \\
\hline $\mathrm{Li}$ & 20.96 & 12.78 & - \\
\hline $\mathrm{C}$ & 18.95 & - & 0.97 \\
\hline $\mathrm{Si}$ & - & - & 94.74 \\
\hline
\end{tabular}

After etching for $300 \mathrm{~s}$, most of the signals from the silicon substrate and weak signals of pure tantalum are present in the spectra, so after $300 \mathrm{~s}$, the film is completely etched away, so the etching rate is about 0.21 $\mathrm{nm} / \mathrm{s}$.
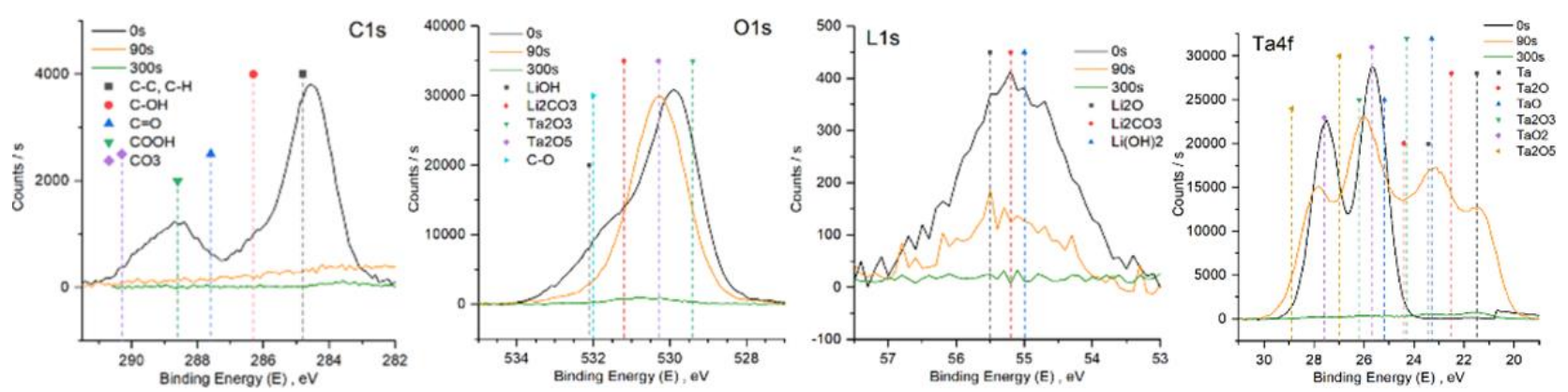

Figure 2 XPS spectra of the Li-Ta-O-1/7 film before and after etching

The X-ray phase analysis pattern (Figure 3) shows two relatively intense peaks characteristic of the Ta structures in the region $27^{\circ}\left(\mathrm{TaO}_{2}\right)$ and $33^{\circ}(\mathrm{TaO})$, as well as one less intense peak in area $62\left(\mathrm{TaO}_{2}\right)$. An ultraintense peak of $33^{\circ}$ is also observed, which can be associated with a defect in the silicon substrate. Comparing the obtained diffractogram with cards from the database of diffractograms PDF, we can conclude that the obtained LiTaO-1/7 film is X-ray amorphous. 


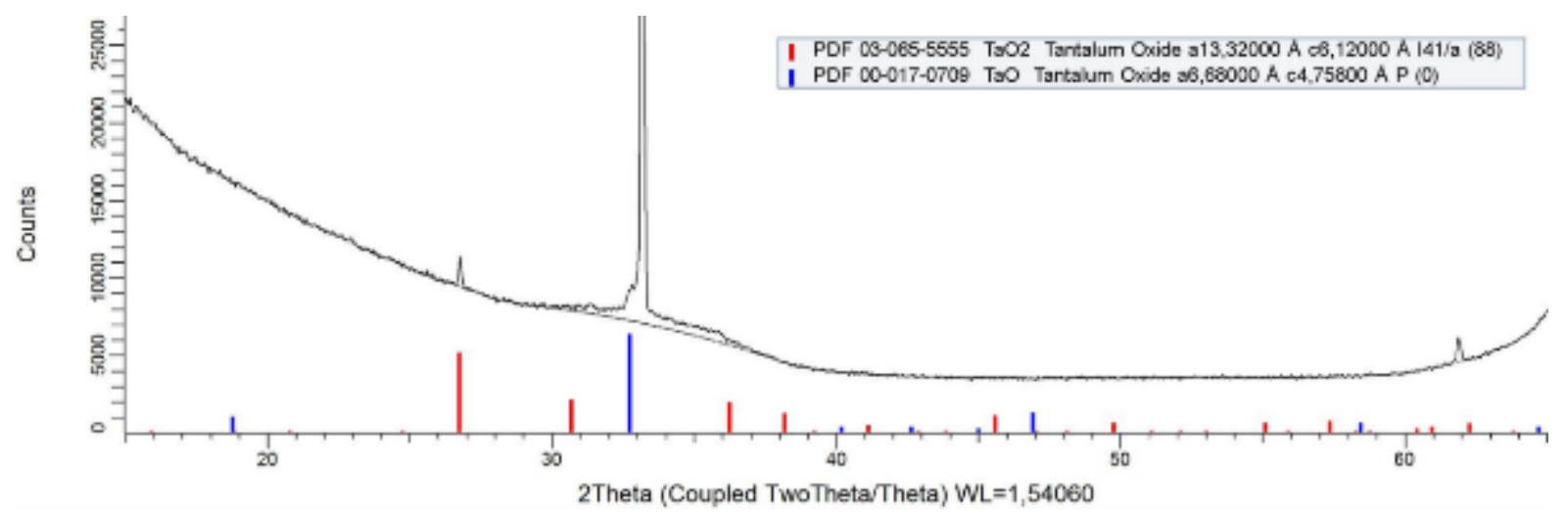

Figure 3 Diffraction pattern of the LiTaO-1/7 sample (Si substrate)

\subsection{Electrochemical tests of Li-Ta-O structures}

The LiTaO-1/2 samples on steel (SS_LiTaO-1/2) with a calculated ratio $\mathrm{Li} / \mathrm{Ta}=1 / 1$ were selected to study the electrochemical characteristics. This ratio was selected based on research results published elsewhere [15], which showed that with an increase of lithium content (from $x=0.32$ to 0.98 in $\mathrm{Li}_{\mathrm{x}} \mathrm{TaO}_{\mathrm{y}}$ ), the lithium-ion conductivity is improved by two orders of magnitude. Based on the results of work [15] and the obtained XPS graphs (Figure 3), we can conclude that with an increase in the Li concentration ( $x=1.73)$, it tends to form harmful bonds with carbon $\left(\mathrm{Li}_{2} \mathrm{CO}_{3}\right)$, hydroxide $\left(\mathrm{Li}(\mathrm{OH})_{2}\right)$ and oxygen $\left(\mathrm{Li}_{2} \mathrm{O}\right)$, instead of being in a free state in the $\left[\mathrm{TaO}_{6}\right]^{-}$crystal lattice. As a result of the formation of such bonds, we lose lithium ions, thereby lowering the overall conductivity of the material.
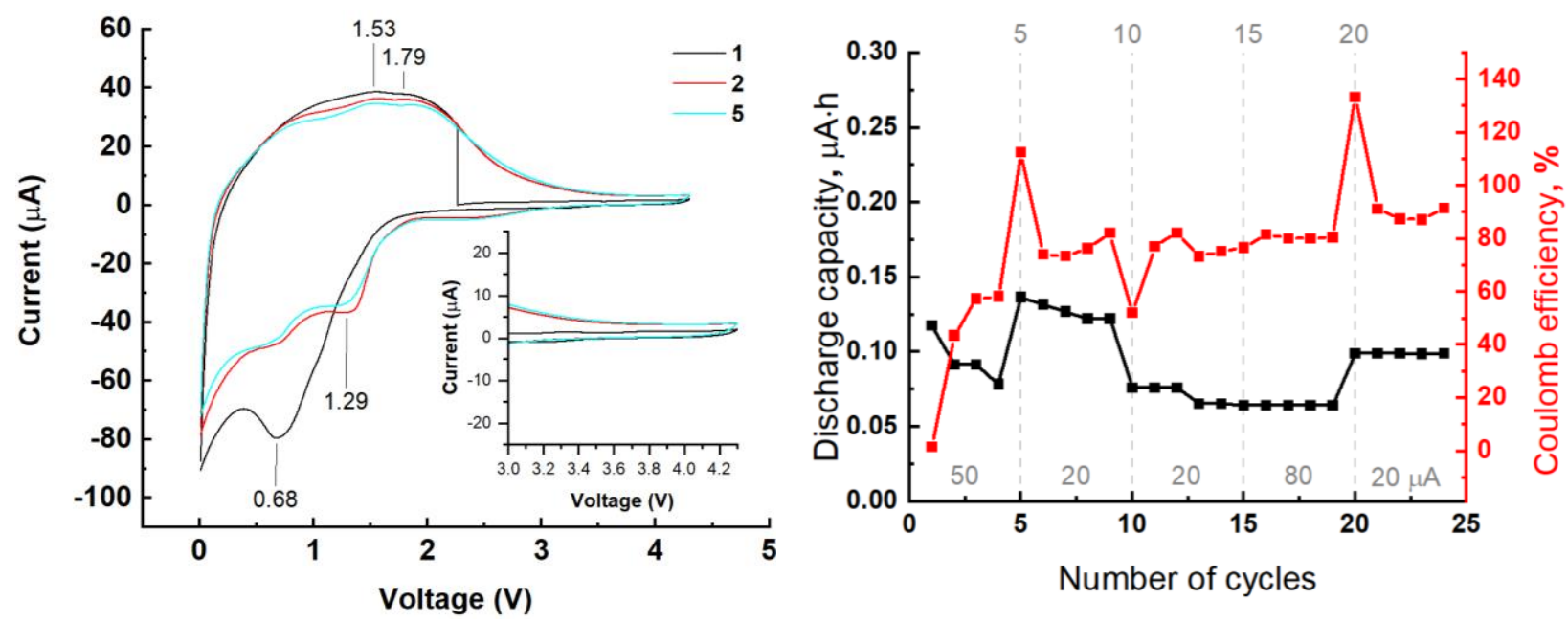

Figure 4 Cyclic voltammetry (CV) curves and results of cyclic tests at different discharge currents

The CV curves of a series of SS_LiTaO-1/2 samples (Figure 4) and coatings of the Ta-O system have a general trend: in the anodic region (3.0-0.1 V), there is current amplification, which is most likely associated with the formation of SEI film [16], and no current increases are observed in the potential cathode range (4.3$3.0 \mathrm{~V}$ - inset in Figure 4). Further cycling at currents from 20 to $80 \mu \mathrm{A} / \mathrm{cm} 2$ in the cathode potential range (4.3$3.0 \mathrm{~V}$ ) showed the presence of a minimum capacity of about 2.0-1.5 $\mu \mathrm{A} \cdot \mathrm{h} \cdot \mu \mathrm{m}-1 \cdot \mathrm{cm}-2(0.14-0.07 \mu \mathrm{A} \cdot \mathrm{h})$, for example in our previous work on the lithium-nickel-silicon oxide cathode study [1], the capacity of the cathode material was $30-20 \mu \mathrm{A} \cdot \mathrm{h} \cdot \mu \mathrm{m}-1 \cdot \mathrm{cm}-2$, so we can consider that this thin film of LiTaO-1/2 does not affect on the final capacity. Thus, the investigated coatings of the LiTaO-1/2 system do not significantly contribute to the electrochemical capacity in the potential cathode range. Therefore, this thin film can be studied like cathodesolid electrolyte systems in the future. 


\section{CONCLUSION}

First, thin films carried out at different synthesis ratios showed different growth rates for LiTaO-1/2, LiTaO-1/3, and LiTaO-1/7: $0.21,0.32$, and $0.63 \mathrm{~nm} /$ supercycle respectively. Secondly, the physicochemical analysis showed: thin films are amorphous; lithium diffuses from the bulk closer to the surface, while tantalum is concentrated in bulk or bottom of coating; traces of carbon (which are absent in bulk) were also found on the surface, which may indicate its interaction with lithium or the formation of organic acids/bases/substances. Finally, electrochemical tests have shown no current amplification in the cathode region (4.3-3.0 V); also, LiTa-O films do not mainly introduce additional capacity, at currents equal $2.0-1.5 \mu \mathrm{A} \cdot \mathrm{h} \cdot \mu \mathrm{m}^{-1} \cdot \mathrm{cm}^{-2}$.

\section{ACKNOWLEDGEMENTS}

\section{The research was supported by the Ministry of Science and Higher Education of the Russian Federation (State Assignment for basic research 0784-2020-0022).}

\section{REFERENCES}

[1] MAXIMOV, M. et al. Atomic layer deposition of lithium-nickel-silicon oxide cathode material for thin-film lithium-ion batteries. Energies. 2020, vol. 13, no. 9.

[2] CHARLES-BLIN, Y. et al. Surface atomic layer fluorination of Li4Ti5O12: Investigation of the surface electrode reactivity and the outgassing behavior in LiBs. Appl. Surf. Sci. Elsevier. 2020, vol. 527, p. 146834.

[3] LI, X. et al. Atomic layer deposition of solid-state electrolyte coated cathode materials with superior high-voltage cycling behavior for lithium ion battery application. Energy Environ. Sci. 2014, vol. 7, no. 2, pp. 768-778.

[4] TALIN, A.A. et al. Fabrication, Testing, and Simulation of All-Solid-State Three-Dimensional Li-lon Batteries. ACS Appl. Mater. Interfaces. 2016, vol. 8, № 47, pp. 32385-32391.

[5] PERSHINA, S. V. et al. Glass-ceramics in Li1+xAlxGe2-x(PO4)3 system: The effect of Al2O3 addition on microstructure, structure and electrical properties. J. Alloys Compd. Elsevier B.V. 2020, vol. 835, p. 155281.

[6] RAJAGOPA,L R., RYU, K.S. A novel Na substituted Li3-xNaxPS4 mixed ions solid electrolyte for all solid-state lithium batteries. Applied Surface Science. Elsevier B.V. 2020, vol. 527, p. 146739.

[7] KIM, G.H. et al. Preparation and Characterization of Sol-Gel-Driven Li x La 3 Zr 2012 Solid Electrolytes and LiCoO 2 Cathodes for All-Solid-State Lithium-Ion Batteries. J. Nanosci. Nanotechnol. 2020, vol. 20, № 11, pp. 7002-7009.

[8] KIM, Y.C. et al. Improving the ionic conductivity of Li1+xAlxGe2-x(PO4)3 solid electrolyte for all-solid-state batteries using microstructural modifiers. Ceram. Int. Techna Group S.r.l. 2020, vol. 46, № 14, pp. 23200-23207.

[9] HAYASHI, D. et al. Synthesis of Li10GeP2S12-type lithium superionic conductors under Ar gas flow. J. Power Sources. Elsevier B. V. 2020, vol. 473, p. 228524.

[10] CUEVAS, A.L. et al. Chemical, optical and transport characterization of ALD modified nanoporous alumina based structures. J. Ind. Eng. Chem. 2020, vol. 91, pp. 139-148.

[11] NGUYEN, V.H. et al. Impact of precursor exposure on process efficiency and film properties in spatial atomic layer deposition. Chem. Eng. J. 2021, vol. 403.

[12] POPOVICH, A.A. et al. Improvement of the cycle life of LiCoO2-based electrodes used in lithium-ion batteries. Russ. J. Appl. Chem. 2015, vol. 88, № 5, pp. 898-899.

[13] KOLCHANOV, D.S. et al. Inkjet Printing of Li-Rich Cathode Material for Thin-Film Lithium-Ion Microbatteries. Energy Technol. 2020, vol. 8, № 3, pp. 1-11.

[14] FEDOROV, P., NAZAROV, D., MEDVEDEV, O. et al. Plasma enhanced atomic layer deposition of tantalum (V) oxide for solid-state batteries. Coatings. 2021.

[15] HU, Y. et al. Ionic conductivity in LixTaOy thin films grown by atomic layer deposition. Electrochim. Acta. Elsevier Ltd. 2020, vol. 361.

[16] KOSHTYAL, Y. et al. Atomic layer deposition of ni-co-o thin-film electrodes for solid-state libs and the influence of chemical composition on overcapacity. Nanomaterials. 2021, vol. 11, № 4. 